\title{
COMPLEMENTARY NIL DOMINATION NUMBER OF A GRAPH
}

\author{
T. TAMIZH CHELVAM AND S. ROBINSON CHELLATHURAI
}

\begin{abstract}
A set $S \subseteq V$ is said to be a complementary nil dominating set of a graph $G$ if it is a dominating set and its complement $V-S$ is not a dominating set for $G$. The minimum cardinality of a $c n d$-set is called the complementary nil domination number of $G$ and is denoted by $\gamma_{\mathrm{cnd}}(G)$. In this paper some results on the complementary nil domination number are obtained.
\end{abstract}

\section{Introduction}

A set $S \subseteq V$ of vertices of a simple graph $G=(V, E)$ is a dominating set if for every vertex $v$ in $V-S$, there exists a vertex $u$ in $S$ such that $v$ is adjacent to u. The minimum cardinality of a dominating set in $G$ is called the domination number of $G$ and is denoted by $\gamma(G)$ [2]. Similarly the maximum cardinality of a minimal dominating set of a graph $G$ is called the upper domination number $\Gamma(G)[2]$. A dominating set $D$ of a graph $G$ is a split dominating set if the induced subgraph $\langle V-D\rangle$ is disconnected. The split domination number $\gamma_{s}(G)$ is the minimum cardinality of a split dominating set [4]. The uniform domination number $\gamma_{u}(G)$ of a graph $G$ is the least positive integer k such that any set with k vertices is a dominating set of $G$ [5]. Let $S \subseteq V$. Then a vertex $v \in S$ is said to be an enclave of $S$ if $N[v] \subseteq S$. The graphs considered here are finite, undirected, without loops or multiple edges and connected with $p$ vertices and $q$ edges. The corona of two graphs $G$ and $H$ is the graph $G o H$ formed from one copy of $G$ and $|V(G)|$ copies of $H$ where the $i^{t h}$ vertex of $G$ is adjacent to every vertex in the $i^{t h}$ copy of $H$. Any undefined terms in this paper may be found in Harary [1].

Definition 1.1. A set $S \subseteq V$ is said to be a $c n d$-set of a graph $G$ if it is a dominating set and its complement $V-S$ is not a dominating set. The minimum cardinality of a $c n d$-set is called the complementary nil domination number of $G$ and is denoted by $\gamma_{\text {cnd }}(G)$.

Corresponding author: T. Tamizh Chelvam.

Received April 12, 2007; revised September 23, 2008.

2000 Mathematics Subject Classification. 05C.

Key words and phrases. Domination number, split domination number, independence number, uniform domination number and enclave. 
Hereafter by a $c n d$-set we mean a complementary nil dominating set. We note that $\gamma_{\text {cnd }}$ sets exist if and only if the graph is not complete. Here after, we assume that $G$ is a non-complete connected graph.

\section{Characterization of complementary nil dominating sets}

First let us prove some basic results on the newly introduced parameter.

Lemma 2.1. Let $S$ be a cnd-set of a graph $G$. Then $S$ contains at least one enclave of $S$.

Proof. Let $S$ be a $c n d$-set of a graph $G$. By Definition 1.1, $V-S$ is not a dominating set, which implies that there exists a vertex $v \in S$ such that $v$ is not adjacent to any vertex in $V-S$ and so $N[v] \subseteq S$.

Remark 2.2. The enclave of a $\gamma_{\mathrm{cnd}}$-set need not be unique. For example, in the graph $C_{5} o C_{3}$, every $\gamma_{\mathrm{cnd}}$-set contains three enclaves.

Proposition 2.3. Let $G$ be a graph and $S$ be a $\gamma_{\mathrm{cnd}}-$ set. If $u$ and $v$ are two enclaves of $S$, then $N[u] \cap N[v] \neq \phi$ and $u$ and $v$ are adjacent.

Proof. Let $u$ and $v$ be two enclaves of $S$. Suppose $N[u] \cap N[v]=\phi$. Then $u$ is an enclave of $S-N(\mathrm{v})$. Clearly $S-N(v)$ is a $c n d$-set of $G$ and $|S-N(v)|<|S|=\gamma_{\text {cnd }}(G)$, which is a contradiction to the minimality of $S$. Hence $N[u] \cap N[v] \neq \phi$. Suppose $u$ and $v$ are non-adjacent, $u \notin N(v)$ and so $S-\{v\}$ contains an enclave $u$ of $S-\{v\}$. Hence $S-\{v\}$ is a $c n d$-set, which is a contradiction to the minimality of S.

Since the complement of an independent set is a dominating set, we get the following.

Proposition 2.4. A cnd-set of a graph $G$ is not an independent dominating set.

The following result is used in the sequel.

Theorem 2.5.([2]) A dominating set $S$ is a minimal dominating set if and only if for each vertex $u \in S$, one of the following two conditions holds:

(a) $u$ is an isolate of $S$,

(b) there exists a vertex $v \in V-S$ for which $N(v) \cap S=\{u\}$.

Theorem 2.6. Let $S$ be a cnd-set of a graph $G$. Then $S$ is minimal if and only if for each vertex $u \in S$ one of the following conditions is satisfied.

(i) $u$ has a private neighbour

(ii) $V-(S-\{u\})$ is a dominating set of $G$.

Proof. Suppose $S$ is minimal. On the contrary if there exists a vertex $u \in S$ such that $u$ does not satisfy any of the given conditions (i) and (ii), then by Theorem 2.5, 
$S_{1}=S-\{u\}$ is a dominating set of $G$. Also by (ii), $V-(S-\{u\})$ is not a dominating set. This implies that $S_{1}$ is a $c n d$-set of $G$, which is a contradiction.

Conversely, suppose that $S$ is a $c n d$-set and for each vertex $u \in S$, one of the two stated conditions holds. We show that $S$ is a minimal $c n d$-set of $G$. On the contrary, we assume that $S$ is not a minimal $c n d$-set. That is, there exists a vertex $u \in S$ such that $S-\{u\}$ is a cnd of $G$. Hence $u$ is adjacent to atleast one vertex in $S-\{u\}$. Also $S-\{u\}$ is a dominating set, every vertex in $V-S$ is adjacent to atleast one vertex in $S-\{u\}$. That is, condition (i) does not hold. Since $S-\{u\}$ is a $c n d$-set, $V-(S-\{u\})$ is not a dominating set. That is condition (ii) does not hold. Therefore there exists a vertex $u \in S$ such that which does not satisfy conditions (i) and (ii), a contradiction to the assumption.

Theorem 2.7. For any graph $G$, every $\gamma_{\mathrm{cnd}}$-set intersects with every $\gamma$-set of $G$.

Proof. Let $S_{1}$ be a $\gamma_{\text {cnd }}$ set and $S$ be a $\gamma$-set of $G$. Suppose that $S_{1} \cap S=\phi$, then $\mathrm{S} \subseteq V-S_{1}, V-S_{1}$ contains a dominating set $\mathrm{S}$. Therefore $V-S_{1}$ itself is a dominating set, which is a contradiction.

Corollary 2.8. In any graph $G$, any two $\gamma_{\mathrm{cnd}}$-sets intersect.

Proposition 2.9. Let $S$ be a cnd-set of a graph $G$. Then there exists a vertex $v$ in $S$ such that $v$ has no private neighbour.

Proof. Since complement of a minimal dominating set is a dominating set, $S$ cannot be a minimal dominating set of $G$. So there exists a vertex $v$ in $S$ such that $v$ has no private neighbour.

\section{Bounds for complementary nil domination number}

In this section, we obtain some bounds for the $c n d$-number of graphs.

Theorem 3.1. For any graph $G, \delta+1 \leq \gamma_{\mathrm{cnd}}(G) \leq \gamma(G)+\delta$.

Proof. Let $S$ be a $\gamma_{c n d}$-set of $G$. Since $V-S$ is not a dominating set, there exists a vertex $v \in S$ which is not adjacent to any of the vertices in $V-S$. Therefore $N[v] \subseteq S$ which implies that $|N[v]| \leq|S|$, that is $d(v)+1 \leq|S|$ and so $\delta+1 \leq \gamma_{\text {cnd }}(G)$. Let $S_{1}$ be a $\gamma i$ set of $G$. Let $u \in V$ such that $d(u)=\delta$. Then atleast one vertex $u_{1} \in N[u]$ such that $u_{1} \in S_{1}$. Now $S_{1} \cup\left(N[u]-\left\{u_{1}\right\}\right)$ is a cnd-set of $\mathrm{G}$, which implies that $\gamma_{\text {cnd }}(G) \leq$ $\left|S_{1} \cup\left(N[u]-\left\{u_{1}\right\}\right)\right| \leq\left|S_{1}\right|+\left|N[u]-\left\{u_{1}\right\}\right|=\gamma+\delta$. Therefore $\delta+1 \leq \gamma_{\text {cnd }}(G) \leq \gamma+\delta$.

In view of the above Theorem 3.1, we have the following corollaries.

Corollary 3.2. For any graph $G$ with $\delta=1, \gamma_{\text {cnd }}(G)=\gamma(G)+1$.

Since, for any graph $\mathrm{G}, \gamma(G) \leq p-\Delta[2]$, we have the following corollary. 
Corollary 3.3. For any graph $G, \gamma_{\mathrm{cnd}}(G) \leq p-\Delta+\delta$.

Observation 3.4.

(i) For any graph $G, \gamma(G)<\gamma_{\text {cnd }}(G)$.

(ii) For any tree $T, \gamma_{\text {cnd }}(T)=\gamma(T)+1$. In particular $\gamma_{\text {cnd }}\left(P_{p}\right)=\gamma\left(P_{p}\right)+1$.

(iii) $\gamma_{\mathrm{cnd}}\left(C_{p}\right)=\left\{\begin{array}{l}\gamma\left(C_{p}\right)+1 \text { if } \mathrm{p} \text { is not a multiple of } 3 \text {. } \\ \gamma\left(C_{p}\right)+2 \text { if } \mathrm{p} \text { is a multiple of } 3 .\end{array}\right.$

(iv) $\gamma_{\mathrm{cnd}}\left(W_{p}\right)=4, p \geq 5$, where $W_{p}$ is a wheel with $p$ vertices.

(v) $\gamma_{\mathrm{cnd}}\left(K_{m, n}\right)=\min \{m, n\}+1$.

(vi) $\gamma_{\mathrm{cnd}}\left(K_{p}-\{e\}\right)=p-1$, where $e$ is an edge in $\mathrm{K}_{\mathrm{p}}$.

(vii) $\gamma_{\text {cnd }}\left(\overline{m K}_{2}\right)=2 m-1$, for $m>1$.

(viii) $2 \leq \gamma_{\text {cnd }}(G) \leq p-1$, for $p \geq 3$.

One can easily prove the following propositions.

Proposition 3.5. For $\mathrm{k}>1$

(i) $\gamma_{\mathrm{cnd}}\left(P_{2} \times P_{k}\right)=\left\{\begin{array}{l}\gamma\left(P_{2} \times P_{k}\right)+1 \text { if } \mathrm{k} \text { is even } \\ \gamma\left(P_{2} \times P_{k}\right)+2 \text { if } k \text { is odd }\end{array}\right.$

(ii) $\gamma_{\mathrm{cnd}}\left(C_{3} \times P_{k}\right)=\gamma\left(C_{3} \times P_{k}\right)+2$.

Proposition 3.6.

(i) $\gamma_{\mathrm{cnd}}\left(\overline{K_{m, n}-e}\right)=m+1$ for $m \leq n$, where $e$ is an edge in $K_{m, n}$.

(ii) $\gamma_{\mathrm{cnd}}\left(\bar{P}_{p}\right)=p-2$ for $p>4$ and $\gamma_{\mathrm{cnd}}\left(\bar{P}_{4}\right)=3$.

(iii) $\gamma_{\text {cnd }}\left(\bar{C}_{p}\right)=p-2$ for $p>4$.

Theorem 3.7. For any graph $G$ with $p>1,\left\lceil\frac{p}{\Delta+1}\right\rceil<\gamma_{\mathrm{cnd}}(G) \leq 2 q-p+1$. Also if $\gamma_{\mathrm{cnd}}(G)=2 q-p+1$ then $G$ is a tree.

Proof. Since $\left\lceil\frac{p}{\Delta+1}\right\rceil \leq \gamma(G)<\gamma_{\mathrm{cnd}}(G)$, the first inequality follows. For any graph $\mathrm{G}, \gamma_{\mathrm{cnd}}(G) \leq p-1=2(p-1)-p+1 \leq 2 q-p+1$.

Also if $\gamma_{\text {cnd }}(G)=2 q-p+1$. Then $2 q-p+1 \leq p-1$ and so $q \leq p-1$. Hence $q=p-1$. Therefore $G$ must be a tree.

Theorem 3.8. Let $G$ be a graph such that both $\mathrm{G}$ and its complement $\bar{G}$ are connected. Then $\gamma_{\mathrm{cnd}}(G)+\gamma_{\mathrm{cnd}}(\bar{G}) \leq(p-1)(p-2)$. Equality holds for $G=P_{4}$.

Proof. By Theorem 3.7, $\gamma_{\text {cnd }}(G) \leq 2 q-p+1$ and $\gamma_{\text {cnd }}(\bar{G}) \leq 2 q-p+1$, then $\gamma_{\text {cnd }}(G)$ $+\gamma_{\mathrm{cnd}}(\bar{G}) \leq 2(q+q)-2(p-1)=p(p-1)-2(p-1)=(p-1)(p-2)$.

Theorem 3.9. Let $G_{1}$ and $G_{2}$ be two connected graphs. Then $\gamma_{\mathrm{cnd}}\left(G_{1} \circ G_{2}\right)=\left|V\left(G_{1}\right)\right|$ $+\delta\left(G_{1} \circ G_{2}\right)$.

Proof. Clearly $V\left(G_{1}\right)$ is a $\gamma\left(G_{1} \circ G_{2}\right)$-set. We choose a vertex $v$ in $G_{1} \circ G_{2}$ such that $d(v)=\delta\left(G_{1} \circ G_{2}\right)$. Then $v$ must be one of the vertices in $V\left(G_{2}\right)$. Let $u$ be the 
vertex in $V\left(G_{1}\right)$ adjacent to $v$. Now $\left(V\left(G_{1}\right)-\{u\}\right) \cup N[v]$ is a $\gamma_{\text {cnd }}$-set of $G_{1} \circ G_{2}$ and $\gamma_{\text {cnd }}\left(G_{1} \circ G_{2}\right)=\left|\left(V\left(G_{1}\right)-\{u\}\right) \cup N[v]\right|=\left|V\left(G_{1}\right)\right|+\delta\left(G_{1} \circ G_{2}\right)$.

In view of Theorem 3.9, we have the following corollaries.

Corollary 3.10. Let $G_{1}$ be any connected graph and $G_{2}$ be a complete graph. Then $\gamma_{\text {cnd }}\left(G_{1} \circ G_{2}\right)=\left|V\left(G_{1}\right)\right|+\left|V\left(G_{2}\right)\right|$.

Corollary 3.11. For any connected graph $H, \gamma_{\mathrm{cnd}}\left(H o K_{1}\right)=|V(H)|+1$.

Theorem 3.12. Let $T$ be a tree with diam $(T)>2$. Then $\gamma_{\mathrm{cnd}}(\bar{T}) \leq p+1-\Delta(T)$. Equality holds for $G=P_{4}$.

Proof. Since $\gamma(\bar{T})=2$, then $\gamma_{\text {cnd }}(\bar{T}) \leq \gamma(\bar{T})+\delta(\bar{T})=2+p-1-\Delta(T)$. Hence $\gamma_{\text {cnd }}(\bar{T}) \leq p+1-\Delta(T)$.

Theorem 3.13. Let $\bar{G}$ be the connected complement of a graph $G$. Then $\gamma_{\mathrm{cnd}}(G)+$ $\gamma_{\text {cnd }}(\bar{G}) \leq \frac{3 p}{2}+1+\delta(G)-\Delta(G)$. Equality holds for $G=P_{4}$.

Proof. From Theorem 3.1, $\gamma_{\text {cnd }}(G) \leq \gamma(G)+\delta(G)$ and $\gamma_{\text {cnd }}(\overline{\mathrm{G}}) \leq \gamma(\overline{\mathrm{G}})+\delta(\overline{\mathrm{G}})=$ $\gamma(\overline{\mathrm{G}})+\mathrm{p}-1-\Delta(\mathrm{G})$. Thus $\gamma_{\text {cnd }}(\mathrm{G})+\gamma_{\text {cnd }}(\overline{\mathrm{G}}) \leq \gamma(\mathrm{G})+\gamma(\overline{\mathrm{G}})+\mathrm{p}-1+\delta(\mathrm{G})-$ $\Delta(\mathrm{G})$. Since $\gamma(G)+\gamma(\bar{G}) \leq \frac{p}{2}+2$ (by Theorem $9.5[2]$ ), from the above, we have $\gamma_{\text {cnd }}(G)+\gamma_{\text {cnd }}(\bar{G}) \leq \frac{p}{2}+2+p-1+\delta(G)-\Delta(G)$. Hence $\gamma_{\text {cnd }}(G)+\gamma_{\text {cnd }}(\bar{G}) \leq \frac{3 p}{2}+1+$ $\delta(G)-\Delta(G)$.

Proposition 3.14. For any tree $T, \gamma_{\mathrm{cnd}}(T)+\in(T) \leq p+1$, where $\in(T)$ is the number of pendant vertices in $T$. Equality holds for $G=K_{1, p-1}$, where $p>2$.

Proof. All the non pendant vertices together a pendant vertex form a complementary nil dominating set. Therefore $\gamma_{\mathrm{cnd}}(T) \leq p-\in(T)+1$ and so $\gamma_{\mathrm{cnd}}(T)+\in(T) \leq p+1$.

Proposition 3.15. For any graph $G, \gamma_{s}(G)<\gamma_{\text {cnd }}(G)$.

Proof. Let $S$ be a $\gamma_{\text {cnd }}$-set of $G$. Then there exists a vertex $v \in S$ such that $N[v] \subseteq S$. Clearly $S-\{v\}$ is a split dominating set and so $|S-\{v\}| \geq \gamma_{s}(G)$. Therefore $\gamma_{\text {cnd }}(G)-1$ $\geq \gamma_{s}(G)$. Hence $\gamma_{s}(G) \leq \gamma_{\text {cnd }}(G)-1<\gamma_{\text {cnd }}(G)$.

Theorem 3.16. For any graph $G, \Gamma(G)+\gamma_{\mathrm{cnd}}(G) \leq p+1$.

Proof. Let $S$ be $\Gamma$-set of $G$. Then there exists a vertex $v \in S$ such that $S-\{v\}$ is not a dominating set of G. But $V-(S-\{v\})$ is a dominating set and so $V-(S-\{v\})$ is a cnd-set. Therefore $|(V-S) \cup\{v\}| \geq \gamma_{\text {cnd }}(G)$. Hence $\Gamma(G)+\gamma_{\text {cnd }}(G) \leq p+1$.

Remark 3.17. In the above Theorem 3.16, the bound $\Gamma(\mathrm{G})+\gamma_{\mathrm{cnd}}(\mathrm{G})=\mathrm{p}+1$ is reachable for the following graphs.

(i) $\mathrm{K}_{\mathrm{p}}-\left\{\mathrm{e}_{1}, \mathrm{e}_{2}, \ldots \ldots, \mathrm{e}_{\mathrm{k}}\right\}$ where $1 \leq k \leq\lfloor p / 2\rfloor, e_{i}$ 's are independent edges, $p>2$.

(ii) All the trees with every non-end vertex is adjacent to atleast one end vertex. 


\section{Particular values of complementary nil domination number}

Theorem 4.1. For any graph $G, \gamma_{\mathrm{cnd}}(\mathrm{G})=2$ if and only if $\gamma(\mathrm{G})=1$ and $\delta=1$.

Proof. Suppose $\gamma_{\text {cnd }}(G)=2$. Then $\gamma(G)<\gamma_{\text {cnd }}(G)=2$, implies $\gamma(G)=1$. Therefore there exits a vertex $u$ in $G$ such that d(u) $=\mathrm{p}-1$. If $\delta>1$, since $\delta+1 \leq \gamma_{\text {cnd }}(G)$, which implies that $\gamma_{\mathrm{cnd}}(G)>2$, which is a contradiction to $\gamma_{\mathrm{cnd}}(G)=2$. Converse follows from Theorem 3.1.

Theorem 4.2. Let $G$ be a graph with $\delta>1$ and $p>3$. Then $\gamma_{\mathrm{cnd}}(\mathrm{G})=\mathrm{p}-1$ if and only if $\delta=\mathrm{p}-2$.

Proof. Suppose that $\gamma_{\mathrm{cnd}}(G)=p-1$. On the contrary, let us assume that $\delta \leq p-3$. Then there exists a vertex $v \in V(G)$ such that $v$ is not adjacent to atleast two vertices say $u, w$ in $V(G)$. Then $V-\{u, w\}$ is a $c n d$-set. So $|V-\{u, w\}| \geq \gamma_{\text {cnd }}(G)$. Hence $\gamma_{\mathrm{cnd}}(G) \leq p-2$, which is a contradiction to the hypothesis. Conversely if $\delta=\mathrm{p}-2$, there exists a vertex $v$ in $G$ with $\mathrm{d}(\mathrm{v})=\mathrm{p}-2$ and $\mathrm{N}[\mathrm{v}]$ is a cnd-set. Therefore $|\mathrm{N}[\mathrm{v}]| \geq \gamma_{\text {cnd }}(\mathrm{G})$, so $\delta+1 \geq \gamma_{\text {cnd }}(\mathrm{G})$. By Theorem 3.1, $\gamma_{\text {cnd }}(\mathrm{G})=\delta+1$. Hence $\gamma_{\text {cnd }}(\mathrm{G})=\mathrm{p}-1$.

\section{Corollary 4.3.}

(i) Let $G$ be a graph with $\delta>1$ and $\mathrm{p}>3$. Then $\gamma_{\mathrm{cnd}}(G)=p-1$ if and only if $G=K_{p}-\left\{e_{1}, e_{2}, \ldots, e_{k}\right\}$ where $1 \leq \mathrm{k} \leq\lfloor\mathrm{p} / 2\rfloor, \mathrm{e}_{\mathrm{i}}$ 's are independent edges.

(ii) Let $G$ be a graph with $\delta=1$ and $\mathrm{p} \geq 5$. Then $\gamma_{\mathrm{cnd}}(\mathrm{G}) \leq \mathrm{p}-2$.

\section{Remark 4.4.}

(i) When $\delta=1$, Theorem 4.2 need not be true, as can be seen from the graph $\mathrm{P}_{4}$.

(ii) In view of Corollary 4.3 , for $\delta=1$, only graphs satisfying $\gamma_{\mathrm{cnd}}(\mathrm{G})=\mathrm{p}-1$ are $P_{3}$ and $P_{4}$.

One can easily prove the following propositions.

Proposition 4.5. Let $G$ be graph with $\delta=1$. Then there exists a $\gamma_{\mathrm{cnd}}$-set which contains atleast one pendant vertex.

Proposition 4.6. Let $T$ be a tree. Then there exists a $\gamma_{\mathrm{cnd}}$-set which contain all the supports and exactly one pendant vertex. Hence $\mathrm{s}+1 \leq \gamma_{\mathrm{cnd}}(\mathrm{G})$, where $\mathrm{s}$ is the number of supports in $T$.

In view of Proposition 4.6, we have the following corollary.

Corollary 4.7. Let $T$ be a tree such that every non-end vertex is adjacent to atleast one end vertex. Then $\gamma_{\mathrm{cnd}}(G)=\mathrm{s}+1$

Theorem 4.8. Let $G$ be a graph with $\operatorname{diam}(G) \geq 3$. Then $\gamma_{\mathrm{cnd}}(G) \leq p-\delta$.

Proof. Let $v \in V$ with $d(v)=\delta$. Since $\operatorname{diam}(G) \geq 3$, there exists a vertex $u \in$ $V-N[v]$ but $u$ is not adjacent to any vertex in $N[v]$. Now, $V-N(v)$ is a dominating set but $\mathrm{N}(\mathrm{v})$ is not a dominating set. Therefore $|V-N(v)| \geq \gamma_{\mathrm{cnd}}(G)$ and $\gamma_{\mathrm{cnd}}(G) \leq p-\delta$. 
For any graph $\mathrm{G}, \gamma_{u}(G)=p-\delta[5]$ and hence we have the following corollary.

Corollary 4.9. Let $G$ be a graph with diam $(G) \geq 3$. Then $\gamma_{\mathrm{cnd}}(\mathrm{G}) \leq \gamma_{\mathrm{u}}(\mathrm{G})$.

Remark 4.10. Corollary 4.9 fails if $\operatorname{diam}(G)=2$. For consider $\mathrm{K}_{2 \mathrm{n}}-\mathrm{X}$ where $\mathrm{X}$ is a 1 -factor in $\mathrm{K}_{2 \mathrm{n}}$. Here $\gamma_{\mathrm{cnd}}=2 \mathrm{n}-1$ for $\mathrm{n}>1$ but $\gamma_{\mathrm{u}}=2$.

Theorem 4.11. Let $G$ be a graph. Then diam $(G)=2$ if and only if $\gamma_{\mathrm{cnd}}(\mathrm{G})=\delta+1$.

Proof. Let $v$ be a vertex in $G$ such that $\mathrm{d}(\mathrm{v})=\delta$, since $G$ is not complete $\delta \leq p-2$. Suppose diam $(G)=2$, every vertex $\mathrm{u} \notin \mathrm{N}(\mathrm{v})$ must be adjacent to some vertex in $\mathrm{N}(\mathrm{v})$. Therefore $\mathrm{N}[\mathrm{v}]$ is a complement nil dominating set of $G$. So $|\mathrm{N}[\mathrm{v}]| \geq \gamma_{\mathrm{cnd}}, \delta+1 \geq \gamma_{\mathrm{cnd}}(\mathrm{G})$. Also by Theorem 3.11, $\delta+1 \leq \gamma_{\text {cnd }}(\mathrm{G})$. Hence $\gamma_{\text {cnd }}(\mathrm{G})=\delta+1$. Convesely, suppose that $\gamma_{\text {cnd }}(\mathrm{G})=\delta+1$. Then there exists a vertex $\mathrm{v} \in \mathrm{G}$ with $\mathrm{d}(\mathrm{v})=\delta$ such that $\mathrm{N}[\mathrm{v}]$ is a $\gamma_{\text {cnd }^{-}}$ set. Therefore every vertex not in $\mathrm{N}(\mathrm{v})$ must be adjacent to some vertex in $\mathrm{N}(\mathrm{v})$ and so $\operatorname{diam}(\mathrm{G})=2$.

In view of the above Theorem 4.11 and $\gamma_{u}(G)=p-\delta[5]$, we have the following corollary.

Corollary 4.12. Let $G$ be a graph. Then $\operatorname{diam}(\mathrm{G})=2$ if and only if $\gamma_{\mathrm{cnd}}(\mathrm{G})+$ $\gamma_{\mathrm{u}}(\mathrm{G})=\mathrm{p}+1$

Proposition 4.13. Let $G$ be a bipartite graph with its complement $\overline{\mathrm{G}}$ connected. Then $\gamma_{\mathrm{cnd}}(\overline{\mathrm{G}})=\mathrm{p}-\Delta(\mathrm{G})$ or $\mathrm{p}-\Delta(\mathrm{G})+1$.

Proof. Let $(X, Y)$ be a partition of $G$. In $\bar{G},\langle X\rangle$ and $\langle Y\rangle$ are complete. Let $u$ be a vertex in $\overline{\mathrm{G}}$ such that $\delta(\overline{\mathrm{G}})=\mathrm{d}(\mathrm{u})$. Without loss of generality we may assume that $u$ is in $\mathrm{X}$. If $\mathrm{N}[\mathrm{u}] \cap \mathrm{Y} \neq \phi$, then $\gamma_{\mathrm{cnd}}(\overline{\mathrm{G}})=|\mathrm{N}[\mathrm{u}]|=\delta(\overline{\mathrm{G}})+1$, since $\delta(\overline{\mathrm{G}})=\mathrm{p}-1-\Delta(\mathrm{G}), \gamma_{\text {cnd }}(\overline{\mathrm{G}})$ $=p-\Delta(G)$. If $\mathrm{N}[\mathrm{u}] \cap \mathrm{Y}=\phi$, then $N[u]$ together with a vertex from $Y$ is a $\gamma_{\text {cnd-set of }}$ $\overline{\mathrm{G}}$. In this case $\gamma_{\mathrm{cnd}}(\overline{\mathrm{G}})=\delta(\overline{\mathrm{G}})+2, \gamma_{\mathrm{cnd}}(\overline{\mathrm{G}})=\mathrm{p}-\Delta(\mathrm{G})+1$.

Theorem 4.14. For any graph $G$, if $\gamma(\mathrm{G})=\frac{\mathrm{p}}{2}$, then $\gamma_{\mathrm{cnd}}(\mathrm{G})=\frac{\mathrm{p}}{2}+1$.

Proof. Suppose $\gamma(\mathrm{G})=\frac{\mathrm{p}}{2}$. Let $S$ be a $\gamma$-set of $G$, which implies $V-S$ is a dominating set with $|V-S|=\frac{p}{2}$. For any vertex $x \in V-S,(V-S)-\{x\}$ is not a dominating set. But $S \cup\{x\}$ is a dominating set and so $S \cup\{x\}$ is a cnd-set. Therefore $|S \cup\{x\}| \geq \gamma_{\text {cnd }}(G)$. So $\gamma(\mathrm{G})+1 \geq \gamma_{\text {cnd }}(\mathrm{G})$. By Theorem 3.1, $\gamma_{\text {cnd }}(\mathrm{G})=\gamma(\mathrm{G})+1$.

Remark 4.15. Converse of the above Theorem 4.14 is not true. For consider $C_{6}$. Here $\gamma_{\mathrm{cnd}}\left(C_{6}\right)=4=\frac{p}{2}+1$, but $\gamma\left(C_{6}\right)=2 \neq \frac{p}{2}$. Assume that $\delta=1$ and $\gamma_{\mathrm{cnd}}(G)=\frac{p}{2}+1$. By Corollary 3.2, $\gamma(G)+1=\gamma_{\text {cnd }}(G)=\frac{p}{2}+1$ and so $\gamma(G)=\frac{p}{2}$.

\section{Acknowledgement}

This work is supported by the University Grants Commission, New Delhi, under the Faculty Improvement Programme for the second author. 


\section{References}

[1] F. Harary, Graph Theory, Addision Wesely, Reading M.A., 1969.

[2] Teresa W. Haynes, Stephen T. Hedetnimi, Peter. J. Slater, Fundamentals of Domination in Graphs, Marcel Decker, 1998.

[3] J. Paulraj Joseph and S. Arumugam, Domination in graphs, International J. Management Systems 11(1995), 177-182.

[4] V.R. Kulli and B.Janakiram, The split domination number of a graph, Graph Theory Notes of New York, New York Academy of Sciences XXXII(1997), 16-19.

[5] S. Arumugam and J. Paulraj Joseph, The uniform domination number of a graph, International J. Management. Systems 11(1995), 111-116.

Department of Mathematics, Manonmaniam Sundaranar University, Tirunelveli 627 012, Tamil Nadu, India.

E-mail: tamche_59@yahoo.co.in

Department of Mathematics, Scott Christian College, Nagercoil 629 003, Tamil Nadu, India.

E-mail: robinchel@rediffmail.com 